\title{
Commentary: The importance of knowing what is beyond the next obstacle
}

\author{
John Bozinovski, MD, MSc
}

In an observational study of 349 patients from one center ${ }^{1}$ embedded in a larger study, ${ }^{2}$ Lin and colleagues sought to address whether coronary artery bypass grafts to targets with chronic total occlusion (CTO) fare differently than grafts to non-CTO targets. A total of 206 patients underwent coronary computed tomography angiography (cCTA) at 6 to 9 years following surgery. Among these patients, 104 had grafting to $\geq 1$ CTO target(s). Grafts to 154 CTO targets were compared with grafts to 569 non-CTO targets. The authors found that grafting a CTO vessel was more likely to fail (adjusted odds ratio [OR], 2.27), as defined by $>50 \%$ stenosis or occlusion of the graft. Arterial grafts, those with higher intraoperative measured flow, side-to-side anastomotic grafts, aspirin use at discharge, and clopidigrel use at discharge were protective of graft patency in CTO targets. But clinical outcomes of death, infarction, and repeat revascularization were not significantly different between the 104 patients with CTO and 102 without CTO. However, the study was not well suited to examine this latter point.

Strengths of the study included multivariable adjustment for patient-level and graft-level characteristics and examining outcomes in subgroups of patients. These included outcomes in those with vein grafts and arterial grafts, outcomes stratified by target vessel territory and outcomes in patients adhering to guideline-directed medical therapy (GDMT) with $\beta$-blockers, statins, and antiplatelet agents. However, stratification in this way further diminishes an already small population of patients. For instance, only $200(27 \%)$ of the grafts were arterial and only $277(38 \%)$ were done in patients adhering to

\footnotetext{
From the Division of Cardiac Surgery, Ohio State University Wexner Medical Center, Columbus, Ohio.

Disclosures: The author reported no conflicts of interest

The Journal policy requires editors and reviewers to disclose conflicts of interest and to decline handling or reviewing manuscripts for which they may have a conflict of interest. The editors and reviewers of this article have no conflicts of interest.

Received for publication April 30, 2020; revisions received April 30, 2020; accepted for publication April 30, 2020; available ahead of print May 16, 2020.

Address for reprints: John Bozinovski, MD, MSc, N816A Doan Hall, 410 West 10th

Ave, Columbus, OH, 43210 (E-mail: jovan.bozinovski@osumc.edu).

J Thorac Cardiovasc Surg 2022;163:1360-1

$0022-5223 / \$ 36.00$

Copyright (c) 2020 by The American Association for Thoracic Surgery

https://doi.org/10.1016/j.jtcvs.2020.04.160
}

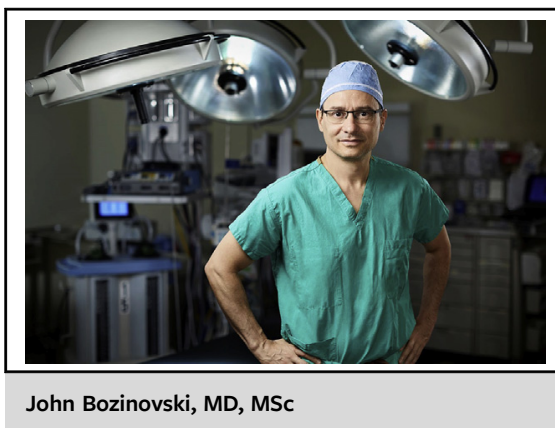

CENTRAL MESSAGE

Chronic total obstruction lesions

are not all alike. Knowing what is

occurring downstream helps

assess the results observed when

bypassing these lesions.

GDMT at the time of cCTA assessment. Moreover, the important characteristic of distal runoff in the target vessel went unexamined.

Nevertheless, in these subgroups, there was no significant difference in arterial graft failure between CTO and nonCTO targets, but there was a significant difference in graft failure for vein grafts. This could reflect selection bias, because surgeons might have been reluctant to perform an arterial graft to a lesion with poor distal runoff, especially in the right coronary artery territory. Grafting right coronary artery territory CTO targets were more likely to fail than non-CTO targets, but left anterior descending and left circumflex territory CTO targets did not fare differently than their non-CTO target comparators. Higher rates of graft occlusion in CTO targets persisted in the $38 \%$ of patients adhering to GDMT.

These graft-level differences did not translate to differences in clinical outcomes, and it is difficult to see how this study will help us make decisions in whether and how to graft CTO targets. Failure of the CTO grafts was not so great as to suggest that grafting was futile or that it caused harm, but neither was it designed to assess the value of CTO grafting. The CORONARY trial from which the patients were drawn was a randomized study comparing on-pump and off-pump surgery in patients age $>70$ or with risk factors for worse outcome. ${ }^{2}$ It was not designed to assess CTO lesions. Neither do we know the viability of the territory to which the grafts were directed or of the downstream flow or collateral flow of the vessel being 
grafted. Graft patency is significantly determined by distal perfusion, as demonstrated by the higher patency rate in grafts with higher intraoperative flow. Without some insight into the distal vascular bed to which these grafts were anastomosed, it is difficult to meaningfully interpret the data.

\section{References}

1. Lin S, Rao C, Yang L, Yang X, Feng W, Sun H, et al. Impact of coronary total oc clusion on graft failure and outcomes of coronary artery bypass grafting. J Thorac Cardiovasc Surg. 2022;163:1349-57.e5

2. Lamy A, Devereaux PJ, Prabhakaran D, Taggart DP, Hu S, Straka Z, et al. Fiveyear outcomes after off-pump or on-pump coronary artery bypass grafting. $N$ Engl J Med. 2016;375:2359-68. 